\title{
Intermittent calorie restriction enhances epithelial-mesenchymal transition through the alteration of energy metabolism in a mouse tumor model
}

\author{
OSAMU KUSUOKA, RINA FUJIWARA-TANI, CHIE NAKASHIMA, KIYOMU FUJII, HITOSHI OHMORI, \\ TAKUYA MORI, SHINGO KISHI, YOSHIHIRO MIYAGAWA, KEI GOTO, \\ ISAO KAWAHARA and HIROKI KUNIYASU
}

Department of Molecular Pathology, Nara Medical University, Kashihara, Nara 634-8521, Japan

Received September 1, 2017; Accepted December 7, 2017

DOI: $10.3892 /$ ijo.2017.4229

\begin{abstract}
The effect of intermittent calorie restriction (ICR) on cancer is controversial. In this study, we examined the effects of ICR and food content in syngeneic BALB/c mice injected with CT26 mouse colon cancer cells. Mice were subjected to 24-h fasting once a week for 4 weeks, and then provided with a control, high-calorie, or trans fatty acid-rich diet. While ICR resulted in increases in tumor weights, metastasis and in the number of cancer stem cells (CSCs) in the tumors or blood of mice fed the control and high-fat diets, it had no effect on body weight after 4 weeks. In particular, we detected increases in the numbers of CSCs in the tumor or blood on the day after starvation, when food overconsumption was detected. Conversely, continuous calorie restriction had no effect on tumor weight, metastasis, or the number of CSCs in tumors or blood. In the post-starvation period, energy metabolism in the tumor was altered from oxidative phosphorylation to glycolysis/lactate fermentation, with the acquisition of the epithelial-mesenchymal transition (EMT) phenotype. Hyperglycemia at the post-starvation period induced the expression of insulin-like growth factor-1, hypoxia-induced factor-1 $\alpha$ and Nanog, as well as the phosphorylation of Stat3. Taken together, these findings suggest that ICR induces an increase in the number of CSCs and enhances EMT by promoting the Warburg/Crabtree effect following post-fasting food overconsumption.
\end{abstract}

\section{Introduction}

Calorie restriction (CR) represents an effective means of regulating aging by inducing a healthy lifespan, as observed

Correspondence to: Professor Hiroki Kuniyasu, Department of Molecular Pathology, Nara Medical University, 840 Shijo-cho, Kashihara, Nara 634-8521, Japan

E-mail: cooninh@zb4.so-net.ne.jp

Key words: calorie restriction, high-fat diet, trans fatty acid in various rodent and primate models (1). By contrast, obesity increases the risk, incidence and mortality rate of patients with various diseases, including breast and prostate cancer (2). Indeed, studies have examined the effects of reducing food consumption by $20-40 \%$ of normal intake (1). Intermittent CR (ICR) is usually performed by restricting energy intake for 1-3 days/week, followed by 'free' eating on non-restriction days. Given that ICR is easier to carry out than traditional CR or continuous CR (CCR), for which energy intake is limited every day, ICR has increased in popularity (3). Moreover, ICR is as effective as traditional CR in achieving weight loss, reducing fat mass and in maintaining the acquired status $(4,5)$.

Weight loss has been shown to decrease the risk of cancer, and to inhibit disease progression and metastasis (6). Whereas traditional CR has been effective in this sense, the anticancer effect of ICR has yet to be proven $(6,7)$. However, it has been proposed that weight cycling, exemplified by CR-induced weight loss followed by weight gain, may decrease the effectiveness of CR, and particularly ICR, on cancer inhibition (7).

Fatty acids, which are found in various food ingredients, influence a wide range of diseases, including cardiovascular disorders, metabolic diseases, such as type-2 diabetes, inflammatory diseases and cancer (8). Evidence indicates that a high-fat diet is associated with aggressive prostate cancer and that $\mathrm{n}-6$ fatty acids enhance breast cancer invasion and metastasis (9). Trans fatty acids (TFAs) represent an established risk factor for cardiovascular diseases, and have been reported to increase the risk of certain types of cancers and to promote cancer cell growth, invasion, anti-apoptotic survival and metastasis (10). In an aim to reduce the incidence of cardiovascular diseases, the United States Food and Drug Administration has ruled that TFAs are not generally recognized as safe for human consumption and must be removed from prepared foods by June 2018 (11).

The overconsumption of saturated fatty acids or TFAs has also been shown to increase the risk of hyperglycemia induced by the enhancement of gluconeogenesis and insulin resistance $(12,13)$. The energy metabolism of cancer cells exhibits a striking feature, referred to the Warburg effect, in which energy production depends predominantly on glycolysis and lactate fermentation, even under aerobic conditions $(14,15)$. 
The Warburg effect is enhanced by the glucose load and also by hyperglycemia induced by high-fat diets or the overconsumption of TFAs (16). As such, alterations in blood sugar levels and energy metabolism in cancer cells associated with ICR is an important subject of study (17). In this study, we therefore sought to characterize the effects of ICR on cancer cells, particularly in combination with a high-fat or high-TFA diet.

\section{Materials and methods}

Cells and reagents. The CT26 mouse colon cancer cell line was a kind gift from Professor I.J. Fidler (MD Anderson Cancer Center, Houston, TX, USA). The cells were cultured in Dulbecco's modified Eagle's medium (DMEM; Wako Pure Chemical Industries, Ltd., Osaka, Japan) supplemented with $10 \%$ fetal bovine serum (Sigma-Aldrich Chemical Co., St. Louis, MO, USA).

DMEM containing $75 \mathrm{mg} / \mathrm{dl}$ of glucose or $150 \mathrm{mg} / \mathrm{dl}$ glucose was generated by mixing glucose-free DMEM with high-glucose DMEM (glucose, $450 \mathrm{mg} / \mathrm{dl}$ ) (both from Wako Pure Chemical Industries, Ltd.). The insulin-like growth factor-1 receptor (IGF-1R) inhibitor, picropodophyllin (working concentration, $500 \mathrm{nM}$ ), and the hypoxia-inducible factor- $1 \alpha(\mathrm{HIF}-1 \alpha)$ inhibitor, chrysin (working concentration, $50 \mu \mathrm{M}$ ), were obtained from Santa Cruz Biotechnology, Inc. (Dallas, TX, USA), while MitoXpress and CellROX green were purchased from Luxcel Biosciences Ltd. (Cork, Ireland) and Thermo Fisher Scientific (Waltham, MA, USA), respectively.

For the measurement of oxygen consumption and reactive oxygen species (ROS) levels, the cells were seeded into 96-well plates (2,000 cells/well) and cultured with regular medium containing MitoXpress $(10 \mu \mathrm{g} / \mathrm{ml})$ overnight or CellROX green $(5 \mu \mathrm{M})$ for $30 \mathrm{~min}$, respectively. After providing the cells with fresh regular medium, the plates were evaluated using a multimode plate reader (Perkin Elmer Japan, Tokyo, Japan) at excitation wavelengths of 340 and $485 \mathrm{~nm}$, and emission wavelengths of 640 and $520 \mathrm{~nm}$ for MitoXpress and CellROX green, respectively.

Animals. A total of 70 male BALB/c mice (4 weeks old, mean body weight, $17.6 \pm 2.4 \mathrm{~g}$ ) were purchased from SLC Japan (Shizuoka, Japan). The animals were maintained in a pathogen-free animal facility in a $12 / 12 \mathrm{~h}$ light/dark cycle in a temperature $\left(22^{\circ} \mathrm{C}\right)$ - and humidity-controlled environment, in accordance with the institutional guidelines approved by the Committee for Animal Experimentation of Nara Medical University, Kashihara, Japan following current regulations and standards of the Japanese Ministry of Health, Labor and Welfare (approval no. 9559).

Animal models. To establish a subcutaneous tumor model, CT26 cancer cells $\left(1 \times 10^{7}\right)$ were inoculated into the scapular subcutaneous tissue of BALB/c mice. Mice were observed for 4 weeks following inoculation. For ICR, the mice were starved for $24 \mathrm{~h}$ (from 8 a.m. on the first day of the week to 8 a.m. on the second day of the week); 4 mice were examined at each time-point. For CCR, the mice were provided access to a low calorie (30\% calorie reduction) diet ad libitum.
The animals were then subjected to the following diets: A control diet [CE-2 (containing 5\% crude fat derived mainly from soy bean oil; CLEA Japan, Inc., Tokyo, Japan)], a high-fat diet [Quickfat (containing 14\% crude fat derived mainly from beef tallow; CLEA Japan, Inc.)], an elaidic acid (EA)-rich diet $[\mathrm{CE}-2+10 \%$ EA (Sigma-Aldrich Chemical Co.)], or a low calorie diet $+10 \%$ EA $[70 \%$ w/w CE- $2+30 \%$ w/w cellulose (Wako Pure Chemical Industries, Ltd.)]. In groups $\mathrm{C}$ and $\mathrm{CI}$, mice were fed the CE-2 diet. In groups F and FI, mice were fed a high-fat diet. In groups E, EI, E-CNT and E-ICR, mice were fed an EA-rich diet. In group E-CCR, mice were fed a low calorie diet. In each group, 5 mice were used. The subcutaneous tumors were excised from the euthanized mice on day 28 to measure tumor weight and examine histologically.

For detecting lung metastasis, indocyanine green-labeled (Dojindo Laboratories, Kumamoto, Japan) mouse anti-epidermal growth factor receptor antibody (EGFR, 1005, sc-03-G; Santa Cruz Biotechnology, Inc.) diluted in PBS $(0.5 \mu \mathrm{g} / 200 \mu \mathrm{l})$ was injected into the caudal vein prior to euthanasia at day 28 . Excised lungs were observed using a Clairvivo OPT in vivo imager (Shimadzu Corp., Kyoto, Japan) (18).

Reverse transcription-polymerase chain reaction ( $R T-P C R)$. In the weekly change studies, the blood was obtained from caudal vein of mice $(n=5)$ at 2 p.m. each day, repetitively. In the energy metabolism experiment, the blood was obtained by cardiac centesis from euthanized mice $(n=5)$ on day 2 of the ICR model, at $6 \mathrm{~h}$ after re-feeding. Total RNA $(1 \mu \mathrm{g})$ was isolated from the blood samples using the PAX gene Blood RNA kit (Qiagen, Venlo, The Netherlands). PCR products were separated on a $2 \%$ agarose gel, visualized with ethidium bromide, and quantified using the QuantiTect Primer assay (Qiagen), according to the manufacturer's instructions. The following primer sets (Sigma Genosys, Ishikari, Japan) were used for amplification: Mouse CD133 (Prom1, accession no. BC028286.1) forward, 5'-gaa aag ttg ctc tgc gaa cc-3' and reverse, 5'-tct caa gct gaa aag cag ca-3'; mouse nucleostemin (NS) $(G \ln 3$, accession no. AY181025.1) forward, 5'-CAG GAT GCT GAC GAT CAA GA-3' and reverse, 5'-TTG ATT GCT CAG GTG ACA GC-3; mouse $\beta$-actin (ActB, accession no. NM_007393.4) forward, 5'-agc cat gta cgt agc cat cc-3' and reverse, 5'-ctc tca gct gtg gtg gtg aa-3'. PCR conditions were set in accordance with the manufacturer's instructions. The number of replicates was 30 cycles $\left(94^{\circ} \mathrm{C} / 1 \mathrm{~min}-64^{\circ} \mathrm{C} / 1 \mathrm{~min}-72^{\circ} \mathrm{C} / 1 \mathrm{~min}\right)$.

Reverse transcription-quantitative PCR (RT-qPCR). The extraction of total RNA was carried out using an RNeasy mini kit (Qiagen), and total RNA (1 $\mu \mathrm{g})$ was synthesized using the ReverTra Ace- $\alpha$-RT kit (Toyobo, Osaka, Japan). qPCR was performed using a StepOne Real-Time PCR system with Fast SYBR ${ }^{\circledR}$-Green Master Mix (Applied Biosystems, Life Technologies, Carlsbad, CA, USA); a relative standard curve quantification method was used for analysis $(18,19)$. PCR was carried out under the conditions recommended by the manufacturer. Hif-1A RT-PCR primers were purchased from Santa Cruz Biotechnology, Inc. $A c t B$ mRNA was amplified as an internal control (GenBank Accession no. NM 001101). Each amplification reaction was evaluated by melting curve analysis. PCR products were visualized by agarose gel electrophoresis with ethidium bromide staining. 
Immunohistochemistry. Consecutive $4-\mu$ m-thick sections were immunohistochemically stained via the immunoperoxidase technique, as previously described (20). Antibodies against mouse CD133 (18470-1-AP; Proteintech, Rosemont, IL, USA), NS (clone E-8, sc-166460; Santa Cruz Biotechnology, Inc.) and mindbomb E3 ubiquitin protein ligase 1 (M7240; MIB1; Ki-67 clone MIB1; Dako Corp., Carpinteria, CA, USA) were used at $0.5 \mu \mathrm{g} / \mathrm{ml}$. Color development was achieved using 3-3'-diaminobenzidine (Dako Corp.). Specimens were counterstained with Meyer's hematoxylin stain (Sigma-Aldrich Chemical Co.) for visualization of the nuclei.

After immunostaining, the number of positively stained nuclei was counted in all slides. To evaluate positivities, 1,000 cells were observed microscopically (BX43; Olympus Lifescience Solution, Tokyo, Japan).

Immunoblot analysis. Whole cell lysates were prepared as previously described (21). Lysates $(50 \mu \mathrm{g})$ were separated by $12.5 \%$ sodium dodecyl sulfate-polyacrylamide gel electrophoresis (SDS-PAGE) and transferred onto nitrocellulose membranes. The membranes were then incubated with primary antibodies specific to Nanog (ab80892; Abcam, Cambridge, UK), Snail (clone C15D3, \#3879; Cell Signaling Technology Japan, Tokyo, Japan), E-cadherin (clone HECD-1, ab1416), vimentin (ab24535) (both from Abcam), Stat3-phosphoTyr705 (clone D3A7, \#9145), or Stat3 (\#9132) (Cell Signaling Technology Japan), followed by peroxidase-conjugated IgG antibodies (\#330 and \#458; MBL, Nagoya, Japan). Anti-tubulin antibody was used to assess the levels of protein loaded per lane (clone DM1A, 05-829; Oncogene Research Products, Cambridge, MA, USA). Immune complexes were visualized using an ECL western blot detection system (Amersham, Aylesbury, UK).

Measurement of blood sugar and lactate levels. In the energy metabolism experiment, blood, obtained by cardiac centesis from euthanized mice $(n=5)$ on day 28 on 2 of the ICR model, at $6 \mathrm{~h}$ after re-feeding was subjected to the measurement of the sugar concentration. The sugar concentration was determined using Medisafe Mini (CR-102; Terumo Corp., Tokyo, Japan) according to the manufacturers' instructions. Lactate levels were measured using the D-Lactate, assay kit (700520; Funakoshi, Tokyo, Japan) according to the manufacturers' instructions.

Enzyme-linked immunosorbant assay (ELISA). The levels of IGF-1 and lactate in whole cell lysates were measured using a mouse IGF1 ELISA kit (Abcam) and an EnzyChrom glycolysis assay kit (BioAssay Systems, Hayward, CA, USA), respectively, according to the manufacturers' instructions.

Statistical analysis. Statistical significance was evaluated by a two-tailed Fisher's exact, Chi-square and unpaired Student's t-tests with Bonferroni correction using InStat software (GraphPad, Los Angeles, CA, USA). Statistical significance was defined as a two-sided P-value $<0.05$.

\section{Results}

Effect of a high-fat diet and intermittent starvation on tumor growth and cancer stem cells (CSCs). First, we assessed the effects of a high-fat (Quickfat) diet on the growth of CT26 colon cell subcutaneous tumors in BALB/c mice, with or without intermittent caloric restriction (ICR) (Fig. 1). The protocol is shown in Fig. 1A. No differences in body weight were observed between the groups (Fig. 1B); however, mice fed the control or high-fat diet had heavier tumors and more nodal metastases when subjected to ICR (groups CI and FI, respectively), compared to the mice not subjected to ICR (groups $\mathrm{C}$ and F, respectively) (Fig. 1C and D). Likewise, the tumors of mice subjected to ICR (groups CI and FI) contained significantly greater numbers of cells positive for CD133, a marker of CSCs, than those in the non-ICR groups (groups $\mathrm{C}$ and F) (Fig. 1E and F).

Effect of ICR on cancer cell stemness. To assess the temporal effects of ICR on the number of CD133+ cells in tumors, we compared $\mathrm{CD}_{133^{+}}$values on day 1 (fasting), day 2 (re-feeding) and day 4 (feeding) in each of the 4 mouse groups (Fig. 2A). Notably, while there was no difference in the number of $\mathrm{CD}_{133^{+}}$cells between the groups, by day 2 , there was a significant increase in the number of $\mathrm{CD} 133^{+}$cells in the mice in groups $\mathrm{CI}$ and FI, compared to those in groups $\mathrm{C}$ and $\mathrm{F}$. However, the number of $\mathrm{CD}_{133^{+}}$cells in the mice in groups $\mathrm{CI}$ and FI decreased to baseline levels by day 4 .

To examine the effect of ICR, we focused on the differences between group F and FI, which were markedly affected by ICR. Via daily comparisons of CD133 mRNA levels, we determined that only the mice in group FI exhibited a transient increase in CD133 expression levels on day 2, which then returned gradually to the control levels (Fig. 2B). Thus, increased CD133 mRNA blood levels might be affected by daily food intake, particularly by over-intake.

Effect of an EA-enriched diet with ICR on tumor growth and $C S C s$. We previously reported that EA (C18:2, trans) modulates CSCs and affects cancer metastability $(22,23)$. Accordingly, in this study, the effects of EA on tumor growth were examined in the same mouse subcutaneous tumor model used above (Fig. 3A). Body weight was indistinguishable between the mice fed an EA-enriched diet with (group EI) or without ICR (group E) or the control diet with (group CI) or without ICR (group C) (Fig. 3B). Tumor weight and cell growth (MIB1 index) were most pronounced in the mice in group EI, followed by those in group E (Fig. 3C and D). Whereas the tumor weight did not differ between the two control groups, the MIB1 index was higher in the mice in $\mathrm{CI}$ than in those in group $\mathrm{C}$. $\mathrm{NS}^{+}$ cells were also most abundant in mice in group EI, followed by those in groups E and CI (Fig. 3E). By contrast, the CD133+ cells were most frequent in mice in group $\mathrm{EI}$, followed by those in groups $\mathrm{CI}$ and $\mathrm{E}$ (Fig. 3F).

EffectofanEA-enricheddietwithICRonmetastasis. To examine the effects of an EA-enriched diet with ICR on metastasis, mice in groups $\mathrm{E}$ and $\mathrm{EI}$ were starved on day 1 (Fig. 4A and B, respectively) and then provided the appropriate diet for the remainder of the week and for 4 weeks. Whereas no significant changes in metastasis were observed in the mice in group E, those in group EI exhibited increased numbers of $\mathrm{NS}^{+}$cells and an increased MIB1 index on day 2. By contrast, there was no change in the numbers of $\mathrm{CD}_{133}{ }^{+}$cells in either group at this time-point. As 
A
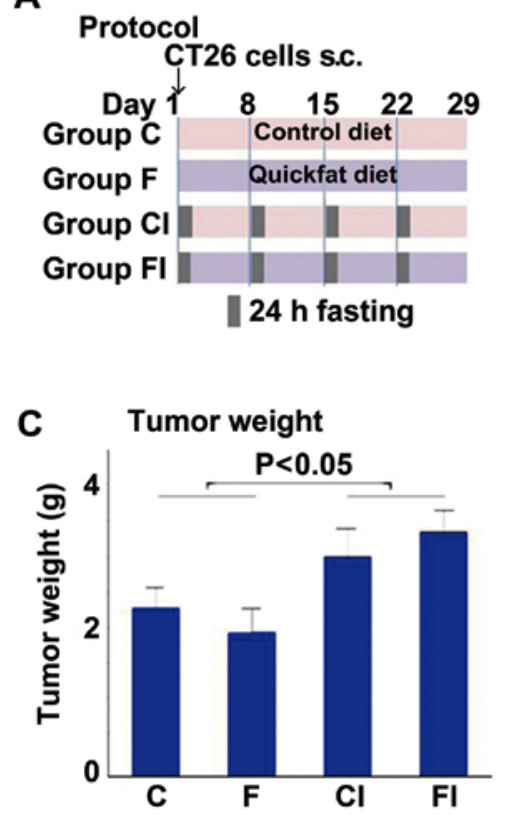

E

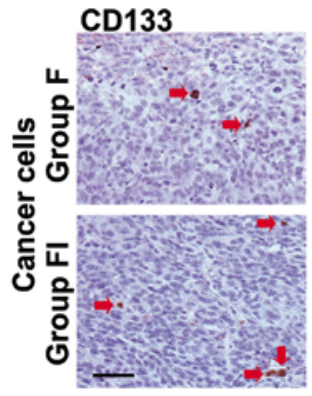

B Body weight on day 29

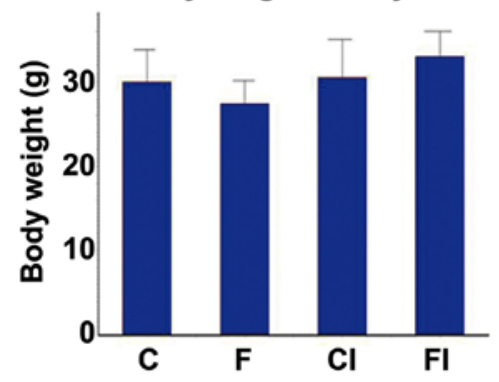

D

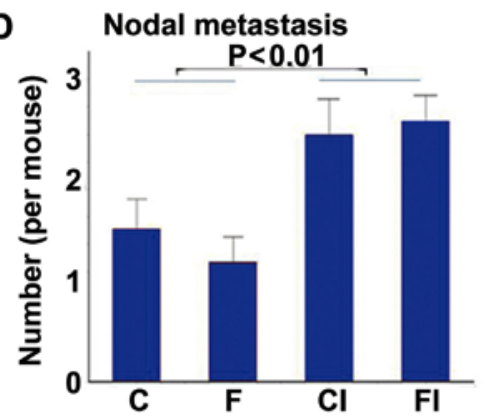

$\mathbf{F}$

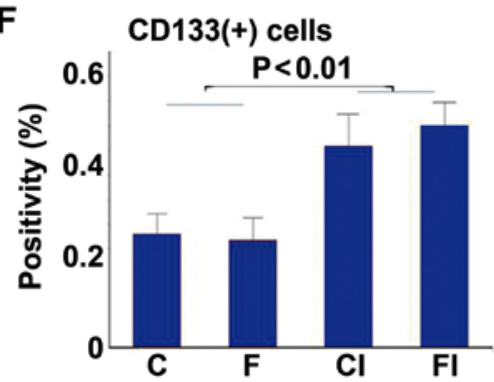

Figure 1. Effect of a high-fat diet and intermittent calorie restriction on tumor growth and cancer stem cells. (A) Protocol for intermittent calorie restriction (ICR) with control and high-fat diets. CT26 cells $\left(1 \times 10^{7}\right)$ were implanted into the scapular subcutaneous tissues of BALB/c mice. Mice were subjected to a 24-h fasting period once each week for 4 weeks. Tumors were excised and examined on day 29. (B) Mouse body weight. (C) Tumor weight. (D) Number of nodal metastases. (E) CD133 immunostaining of tumors from mice in groups F (high-fat diet) and FI (high-fat diet with ICR). CD133-positive cells are indicated by red arrows. Scale bar, $50 \mu \mathrm{m}$. (F) Number of CD133 ${ }^{+}$cells in tumors of mice in each group. Group C, controls not subjected to ICR; group CI, controls subjected to ICR.

shown in Fig. 4C, the mice subjected to ICR (groups CI and EI) exhibited a surge in NS mRNA levels in the blood on the day after starvation. At 4 weeks after inoculation, micrometastatic foci were detected in the lungs by immunofluorescence using anti-EGFR antibody (18). The intensity of fluorescence increased in the following order: $\mathrm{C}<\mathrm{CI}<\mathrm{E}<\mathrm{EI}$ (Fig. 4D). Specifically, the intensities in CI and EI groups increased to levels 1.8- and 6-fold greater than those observed in groups $\mathrm{C}$ and $\mathrm{E}$, respectively.

Comparison of EA-associated pro-tumoral effect between $I C R$ and CCR. Subsequently, we examined the effects of ICR on the pro-tumoral effect of EA in comparison with CCR (Fig. 5). We prepared the low calorie diet by mixing the standard diet (CE-2) with edible cellulose at a 7:1 ratio. This food therefore contained $70 \%$ of the calories of the CE-2 diet. As shown in the protocol (Fig. 5A), the mice were divided into 3 groups as follows: the no calorie restriction with $10 \%$ EA (E-CNT), ICR with 10\% EA (E-ICR) and CCR with $10 \%$ EA (E-CCR) groups. While there were no differences in body weight between the 3 groups on day 29, the mice in the E-ICR group exhibited increased tumor weights and MIB1 index scores, compared to those in the E-CNT or E-CCR groups (Fig. 5C and D).

The examination of daily caloric intakes indicated that mice in the E-CCR group ate a smaller volume of food to reach $80 \%$ of the calorie intake of mice in the E-ICR group on non-starvation day (the expected calorie intake was $70 \%$ in E-CCR group) (Fig. 5E). Specifically, the mice in the E-CCR group consumed $83 \%$ of the calories as those in the E-ICR group weekly. However, this over-intake of food resulted in an enhanced EA intake $(117 \%)$ in mice provided with E-CCR (Fig. 5F), compared to those provided the E-ICR diet.

To confirm the observed surge in stem cell marker expression in the blood (Figs. 2 and 4), we measured the mRNA expression levels of NS in the blood samples from each mouse on day 2 (Fig. 5G). Notably, the mice in the E-ICR group, but not those in the E-CCR group, exhibited an enhanced NS mRNA expression. Likewise, lung micrometastasis was increased in mice provided the E-ICR diet, but not in those given the E-CCR diet (Fig. 5H).

Effect of post-starvation overconsumption on energy metabolism, stemness and the EMT phenotype in CT26 cells. To 
Table I. Comparison of tumors in starvation phase with those in the post-starvation (over-intake) phase.

\begin{tabular}{|c|c|c|c|c|c|c|}
\hline & \multicolumn{3}{|c|}{ Control diet } & \multicolumn{3}{|c|}{$10 \%$ EA diet } \\
\hline & Starvation & Over-intake & P-value ${ }^{a}$ & Starvation & Over-intake & $\mathrm{P}$-value ${ }^{\mathrm{a}}$ \\
\hline Blood sugar (mg/dl) & $78 \pm 8.2$ & $148 \pm 16$ & $<0.0001$ & $84 \pm 9.1$ & $167 \pm 18.4$ & $<0.0001$ \\
\hline MIB1 positivity (\%) & $48 \pm 3.1$ & $65 \pm 5.8$ & $<0.001$ & $62 \pm 6.3$ & $88 \pm 7.4$ & $<0.001$ \\
\hline NS positivity (\%) & $44 \pm 3.2$ & $62 \pm 7.1$ & $<0.001$ & $55 \pm 6.4$ & $76 \pm 6.2$ & $<0.001$ \\
\hline $\mathrm{O}_{2}$ consumption $(\mathrm{kpiu})^{\mathrm{b}}$ & $7.5 \pm 0.51$ & $4.5 \pm 0.25$ & $<0.0001$ & $8.9 \pm 0.59$ & $4.2 \pm 0.18$ & $<0.0001$ \\
\hline Lactate $(\mathrm{pM})$ & $2.1 \pm 0.31$ & $7.2 \pm 0.60$ & $<0.0001$ & $2.4 \pm 0.15$ & $8.7 \pm 0.66$ & $<0.0001$ \\
\hline $\operatorname{ROS}(\%)^{\mathrm{c}}$ & $100 \pm 12$ & $57 \pm 7$ & $<0.0001$ & $121 \pm 11$ & $63 \pm 5$ & $<0.0001$ \\
\hline
\end{tabular}

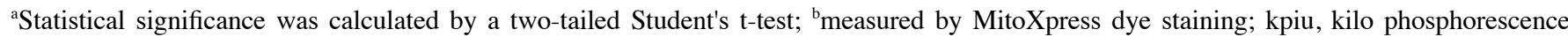
intensity; creactive oxygen species levels were measured by CellROX Green dye staining. Fluorescence intensity is standardized by that of control diet-starvation group, which is set to $100 \%$. Anti-Kie-67 protein antibody clone MIB1; NS, nucleostemin; ROS, reactive oxygen species.
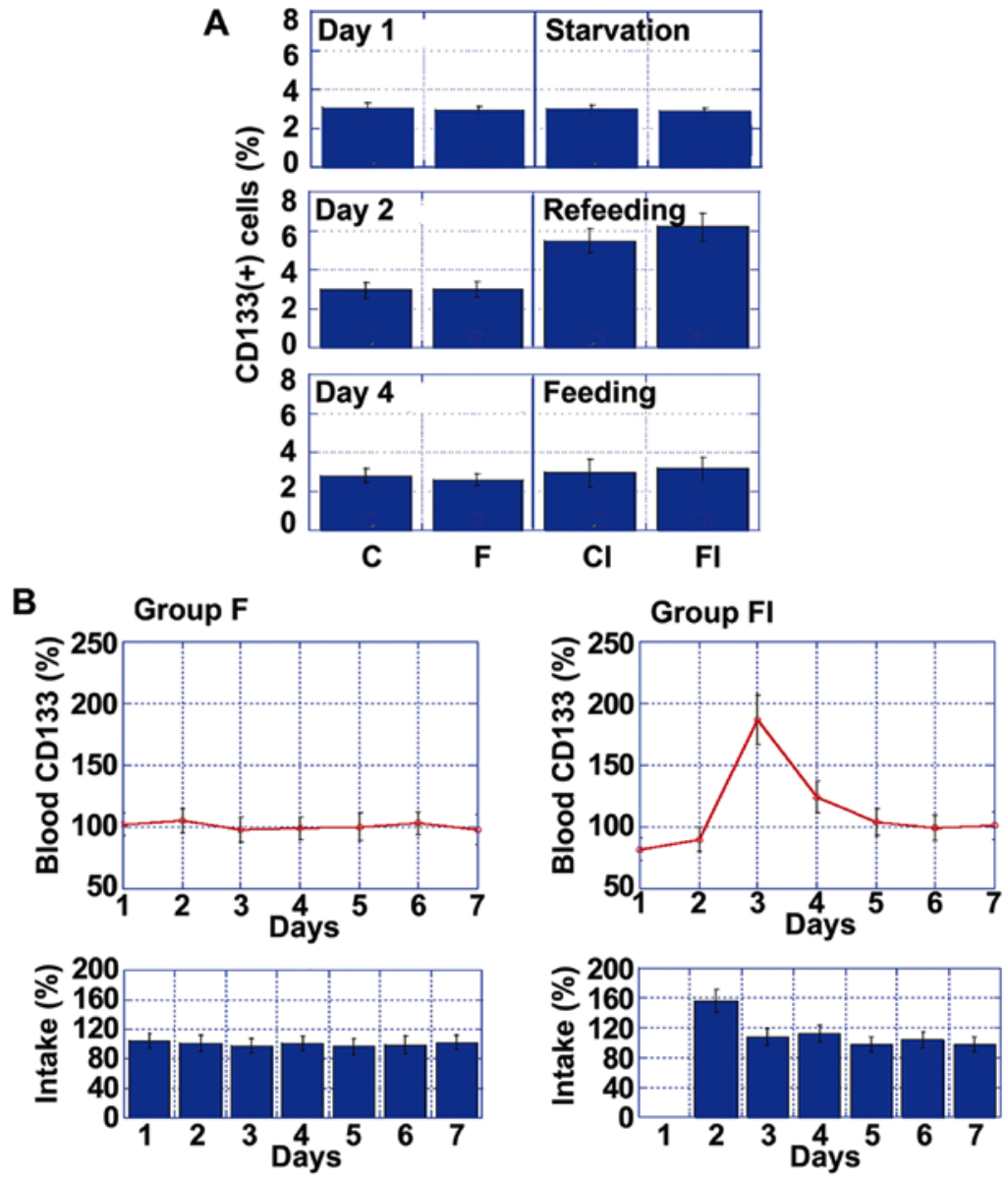

Figure 2. Temporal changes in CD133 expression. (A) Percentage of CD133+ cells on day 1 (fasting), day 2 (re-feeding) and day 4 (feeding). (B) Time-course analysis of CD133 mRNA levels in the blood and food intake in groups F (high-fat diet) and FI (high-fat diet with ICR). mRNA levels and food calorie intake were standardized to those on day 1 for group F, which were set as $100 \%$. Error bars indicate the means \pm standard deviations. Group C, controls not subjected to intermittent calorie restriction (ICR); group CI, controls subjected to ICR.

examine the energy metabolism and stemness phenotypes of CT26 tumors in the post-starvation phase, tumors harvested between the starvation and post-starvation phase were compared in mice fed the control or $10 \%$ EA diet (Table I). In both groups, blood sugar concentrations were elevated 2-fold in the post-starvation (over-intake) phase, compared to the starvation phase. Likewise, the levels of cell proliferation (MIB1) and stemness (NS) markers were also increased in the post-starvation phase. Notably, while energy was primarily produced via oxidative phosphorylation (OXPHOS) during starvation, cells exhibited a switch to glycolysis/lactate fermentation (GL/LF)-mediated energy production during the post-starvation phase, which was accompanied by a decrease in ROS production. 


\section{A Protocol}
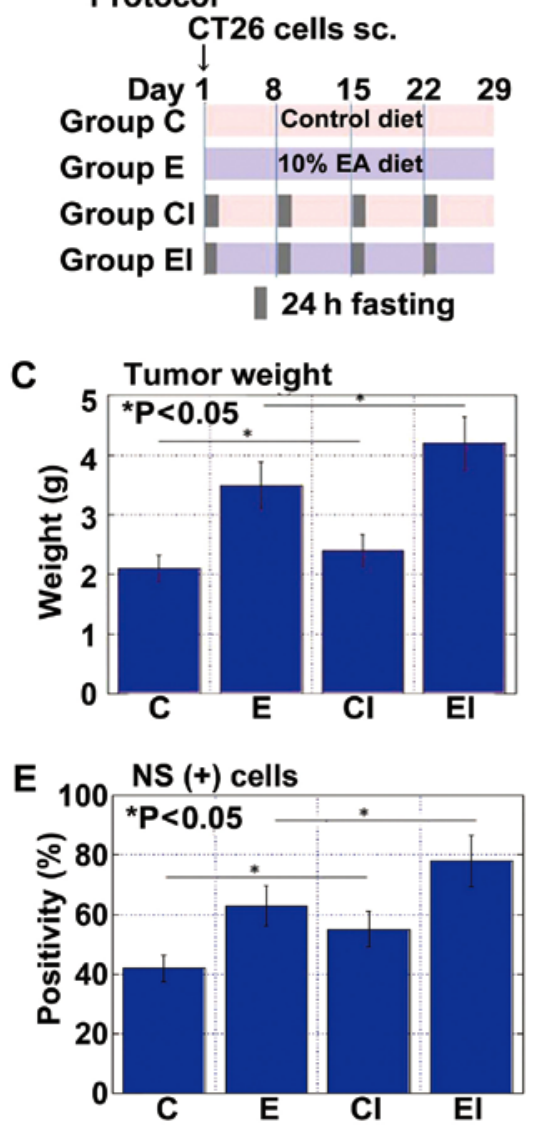
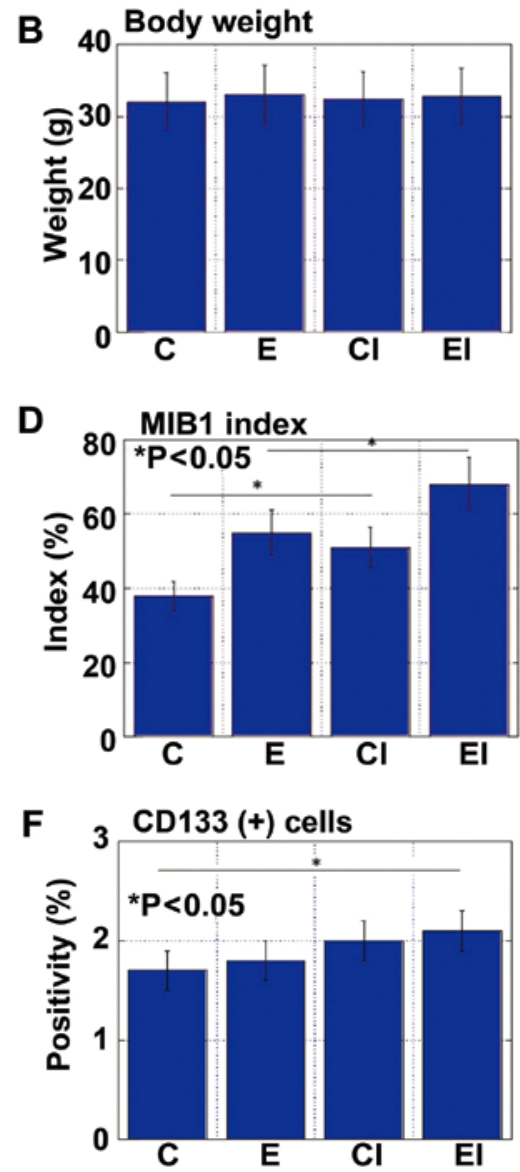

Figure 3. Effect of an elaidic acid (EA)-enriched diet with intermittent calorie restriction (ICR) on tumor growth and cancer stem cells. (A) Protocol for ICR with a control and EA-enriched diet. CT26 cells $\left(1 \times 10^{7}\right)$ were implanted into the scapular subcutaneous tissues of BALB/c mice. Mice were subjected to a 24-h fasting period once each week for 4 weeks. Tumors were excised and examined on day 29. (B) Mouse body weight. (C) Tumor weight. (D) MIB1 index. (E) Percentage of nucleostamin-positive $\left(\mathrm{NS}^{+}\right)$cells. $(\mathrm{F})$ Percentage of $\mathrm{CD} 133^{+}$cells. Error bars indicate the means \pm standard deviations. Group C, controls not subjected to ICR; group CI, controls subjected to ICR; group E, mice given EA-rich diet not subjected to ICR; group EI, mice given EA-rich diet subjected to ICR.

Table II. Comparison of CT26 cells cultured under hypoglycemic and normoglycemic conditions.

\begin{tabular}{lccc}
\hline & \multicolumn{2}{c}{$\begin{array}{c}\text { Glucose concentration } \\
(\mathrm{mg} / \mathrm{dl})\end{array}$} & \\
\cline { 2 - 3 } & 75 & 150 & P-value \\
& & $70 \pm 6.2$ & $<0.01$ \\
\hline MIB1 positivity (\%) & $45 \pm 4.9$ & $58 \pm 6.1$ & $<0.05$ \\
NS positivity (\%) & $40 \pm 4.1$ & $5.78 \pm 0.302$ & $<0.001$ \\
$\mathrm{O}_{2}$ consumption (kpiu) & $8.75 \pm 0.626$ & 4.01 & $<0.001$ \\
Lactate (pM) & $1.9 \pm 0.22$ & $8.1 \pm 0.71$ & $<0.01$ \\
ROS (\%) & $100 \pm 9$ & $62 \pm 7$ & \\
\hline
\end{tabular}

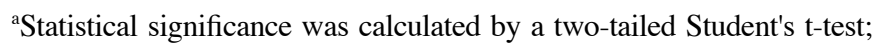
bmeasured by MitoXpress dye staining; kpiu, kilo phosphorescence intensity; 'reactive oxygen species levels were measured by CellROX Green dye staining. Fluorescence intensity is standardized by that of control diet-starvation group, which is set to $100 \%$. Anti-Kie-67 protein antibody clone MIB1; NS, nucleostemin; ROS, reactive oxygen species.

To confirm the effects of sequential increases in blood glucose concentrations on energy metabolism and stemness, the CT26 cells were cultivated in medium containing
$75 \mathrm{mg} / \mathrm{dl}$ glucose, followed by medium containing $150 \mathrm{mg} /$ dl glucose (Table II). These glucose concentrations were selected to simulate those observed in the blood of mice, as shown in Table I. The CT26 cells cultured in the presence of $150 \mathrm{mg} / \mathrm{dl}$ glucose exhibited increased cell proliferation, stemness and GL/LF, and decreased OXPHOS and ROS production, compared to those cultivated in the presence of $75 \mathrm{mg} / \mathrm{dl}$ glucose.

Subsequently, CT26 tumors in the animal model and CT26 cells in in vitro treatment were subjected to immunoblot analysis to compare the expression levels of EMT- and stemness-specific proteins between the starvation phase and post-starvation phase and between cultivation with 75 and $150 \mathrm{mg} / \mathrm{dl}$ glucose, respectively (Fig. 6A and B). The tumors harvested during the post-starvation phase or the cells after cultivation in medium containing $150 \mathrm{mg} / \mathrm{dl}$ glucose exhibited increases in Nanog expression (stemness marker), and in Snail and vimentin expression (EMT markers), as well as decreased E-cadherin expression and increased phosphorylation of Stat3, compared to the cells harvested during the fasting period or after cultivation in the presence of $75 \mathrm{mg} / \mathrm{dl}$ glucose. Moreover, these cells exhibited an increase in the mRNA expression of HIF- $1 \alpha$ and the protein expression of IGF-1 (Fig. 6C and D).

To examine the signaling pathway associated with the alterations observed during the post-starvation phase, the CT26 cells 
Table III. Effects of the inhibitors of IGF-1R and HIF-1 $\alpha$ on energy metabolism in CT26 cells.

\begin{tabular}{|c|c|c|c|c|c|}
\hline Glucose concentration (mg/dl) & 150 & 150 & P-value ${ }^{a}$ & 150 & P-value ${ }^{a}$ \\
\hline Inhibitor & None & IGF-1R & & HIF- $1 \alpha$ & \\
\hline MIB1 positivity (\%) & $71 \pm 6.5$ & $45 \pm 4$ & $<0.01$ & $42 \pm 3.1$ & $<0.01$ \\
\hline NS positivity (\%) & $61 \pm 6.3$ & $42 \pm 4.4$ & $<0.05$ & $40 \pm 3.5$ & $<0.01$ \\
\hline $\mathrm{O}_{2}$ consumption $(\mathrm{kpiu})^{\mathrm{b}}$ & $4.67 \pm 0.298$ & $7.44 \pm 0.752$ & $<0.001$ & $7.58 \pm 0.788$ & $<0.001$ \\
\hline Lactate $(\mathrm{pM})$ & $7.9 \pm 0.84$ & $2.2 \pm 0.24$ & $<0.001$ & $2.0 \pm 0.25$ & $<0.001$ \\
\hline $\operatorname{ROS}(\%)^{\mathrm{c}}$ & $64 \pm 7$ & $99 \pm 10$ & $<0.01$ & $103 \pm 11$ & $<0.01$ \\
\hline
\end{tabular}

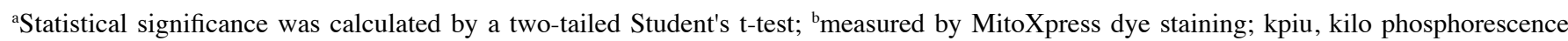
intensity; creactive oxygen species levels were measured by CellROX Green dye staining. Fluorescence intensity is standardized by that of control diet-starvation group, which is set to $100 \%$. Anti-Kie-67 protein antibody clone MIB1; NS, nucleostemin; ROS, reactive oxygen species; IGF-1R, insulin-like growth factor-1 receptor; HIF-1 $\alpha$, hypoxia-inducible factor-1 $\alpha$.
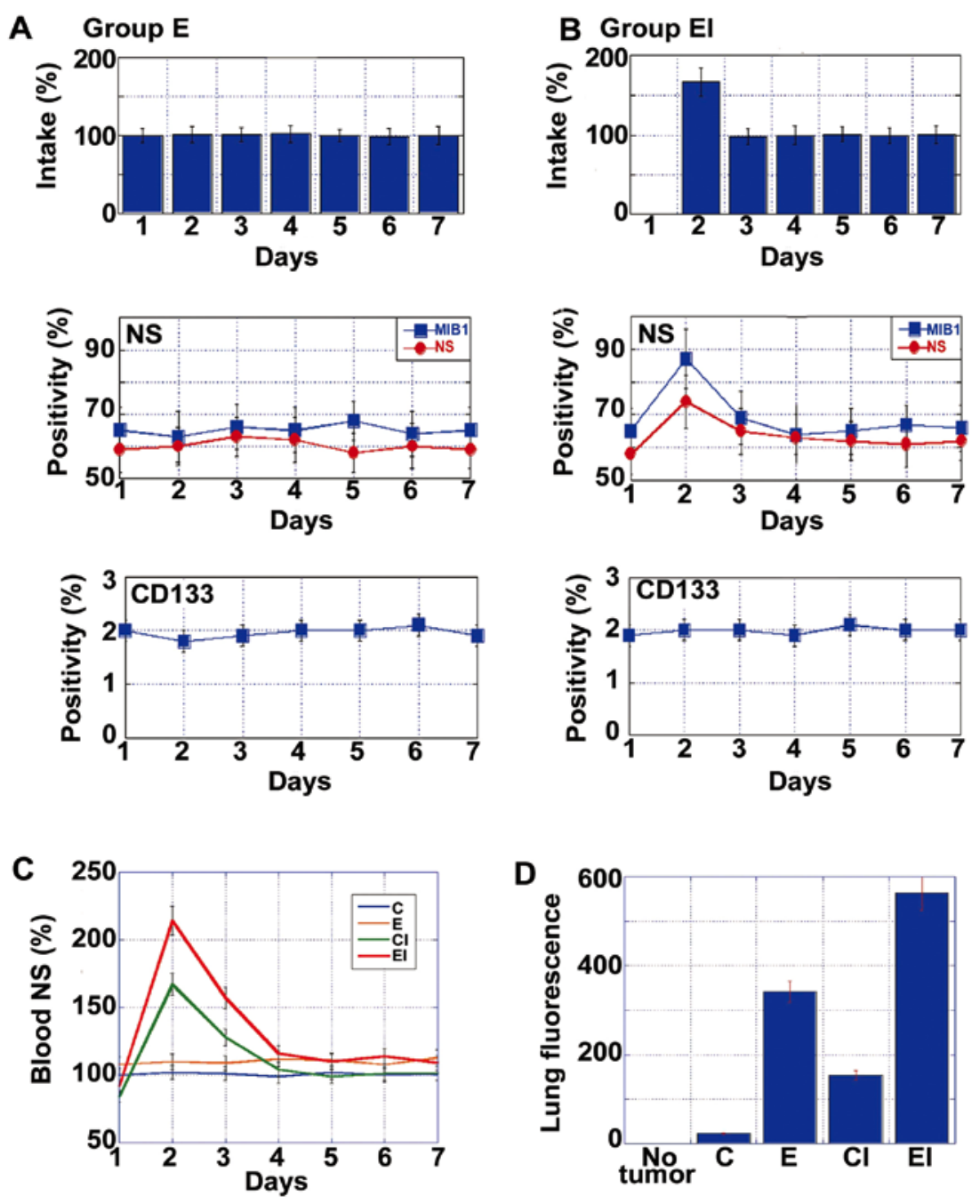

Figure 4. Effect of an EA-enriched diet with intermittent calorie restriction (ICR) on cancer metastasis. (A) Temporal changes in food intake and the number of nucleostamin-positive $\left(\mathrm{NS}^{+}\right)$and $\mathrm{CD} 133^{+}$cells in group E (EA-rich diet without ICR) tumors. (B) Temporal changes in food intake and the number of NS ${ }^{+}$and $\mathrm{CD}_{133^{+}}$cells in group EI (EA-rich diet with ICR) tumors. Food calorie intake was standardized to that on day 1 for group E, which was set as $100 \%$. Positivity of NS or CD133 was calculated as the percentage of positively stained cells among 200 cells observed. (C) Time-course analysis of NS mRNA levels in the blood. (D) Micrometastasis in the lung on day 29. Cancer cells were detected by ICG-labeled anti-EGFR antibody and measured using an in vivo imager. Error bars indicate the means \pm standard deviations. Group C, controls not subjected to ICR; group CI, controls subjected to ICR; group E, mice given EA-rich diet not subjected to ICR; group EI, mice given EA-rich diet subjected to ICR.

were treated with inhibitors of IGF-1R or HIF-1 $\alpha$ (Table III). Both treatments, respectively, abrogated the effects observed in
CT26 cells cultured in medium containing $150 \mathrm{mg} / \mathrm{dl}$ glucose, following medium containing $75 \mathrm{mg} / \mathrm{dl}$ glucose. 
A

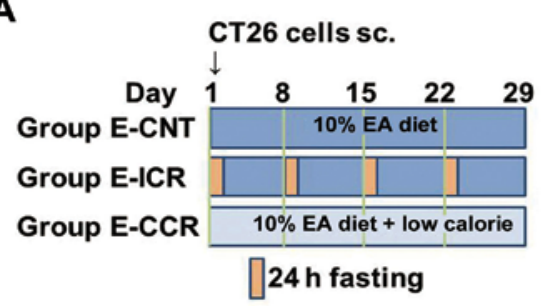

C

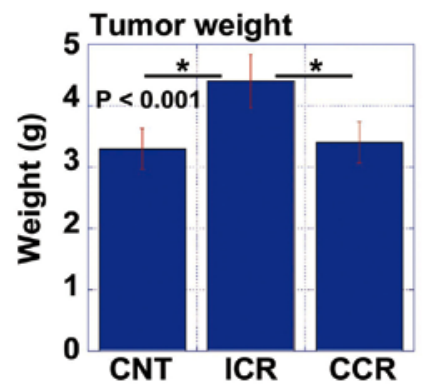

E

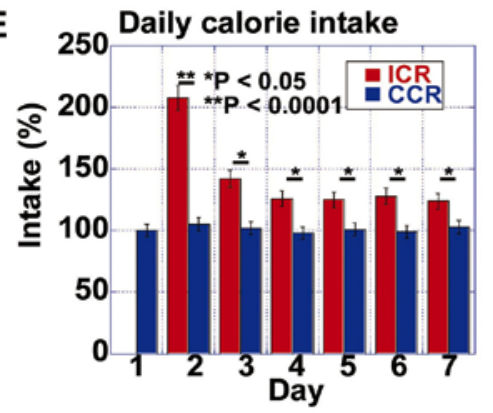

G

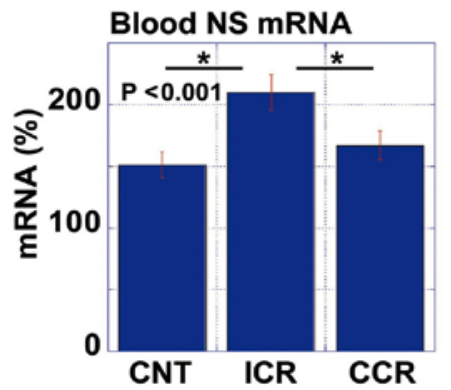

B

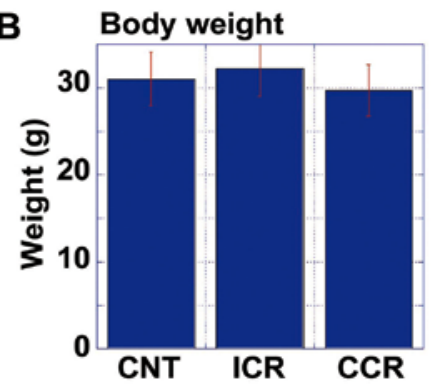

D
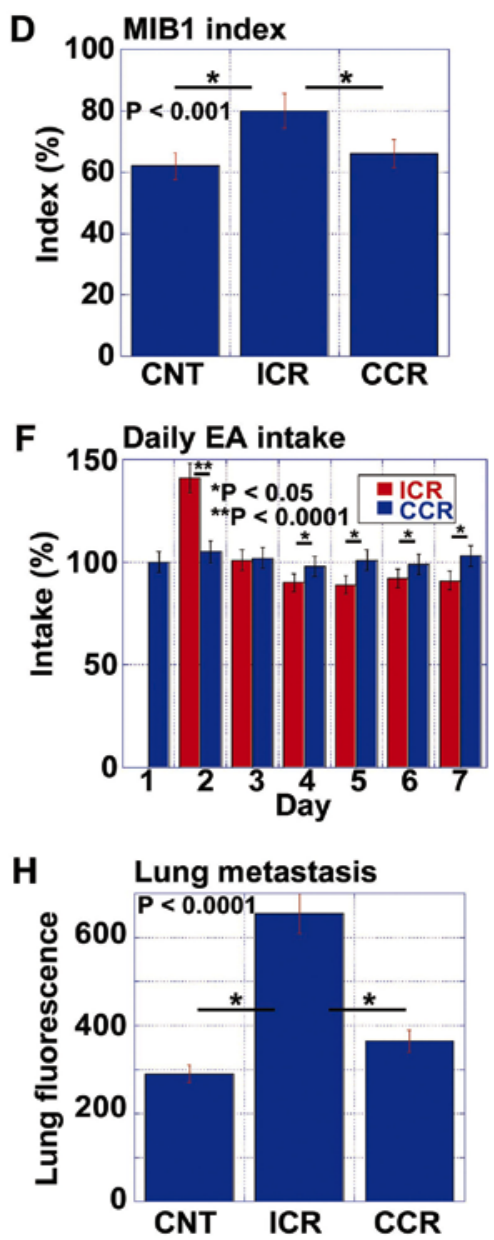

Figure 5. Comparison of elaidic acid (EA)-associated pro-tumoral effect between intermittent calorie restriction (ICR) and continuous calorie restriction (CCR). (A) Protocol for assessing pro-tumoral effect of EA on mice not subjected to calorie restriction (E-CNT), with intermittent calorie restriction (E-ICR), or with continuous calorie restriction (E-CCR). CT26 cells $\left(1 \times 10^{7}\right)$ were implanted into the scapular subcutaneous tissues of BALB/c mice. For ICR, the mice were subjected to a 24-h fasting period once each a week for 4 weeks. CCR was achieved by feeding a low calorie diet [70\% CE-2 (control) $+30 \%$ cellulose (w/w)]. (B-D) Body weight, tumor weight and tumor proliferation (MIB1 index), respectively for each of the 3 groups. (E and F) Time-course analysis of calorie intake and EA intake, respectively among the EA-ICR and EA-CCR groups. (G) Nucleostemin (NS) mRNA levels in the blood on day 2. (H) Micrometastasis in the lungs on day 29. Cancer cells were detected by ICG-labeled anti-claudin 4 antibody and measured using an in vivo imager. Error bars indicate the means \pm standard deviations.

\section{Discussion}

In this study, we examined the effects of ICR on tumor growth using a mouse syngeneic subcutaneous tumor model. Our findings demonstrated that instead of having a tumor suppressive effect, ICR promoted tumor growth and increased the number of CSCs. Such a pro-tumoral effect was suggested to depend on the induction of a starvation-overeating cycle.

In designing the ICR experiments, starvation for $1 \mathrm{day} /$ week was expected to decrease dietary calorie intake by $14 \%$. However, overeating after starvation abrogated CR, and dietary calorie loss was only $1.5 \%$. This was clearly confirmed by the lack of any difference in body weight between the mice subjected to ICR and those not subjected to ICR. Strictly speaking, our experimental design therefore achieved dietary restriction, but not CR. Dietary restriction is defined as intermittent fasting without CR (1) and has been shown to effectively prolong lifespan with minimal adverse effects (1), as well as to ameliorate obesity and type-2 diabetes $(24,25)$.

We also compared our ICR mouse model with a CCR mouse model. While CCR model achieved an $\sim 80 \%$ loss in dietary calorie intake each day, the mice in the CCR group 
A
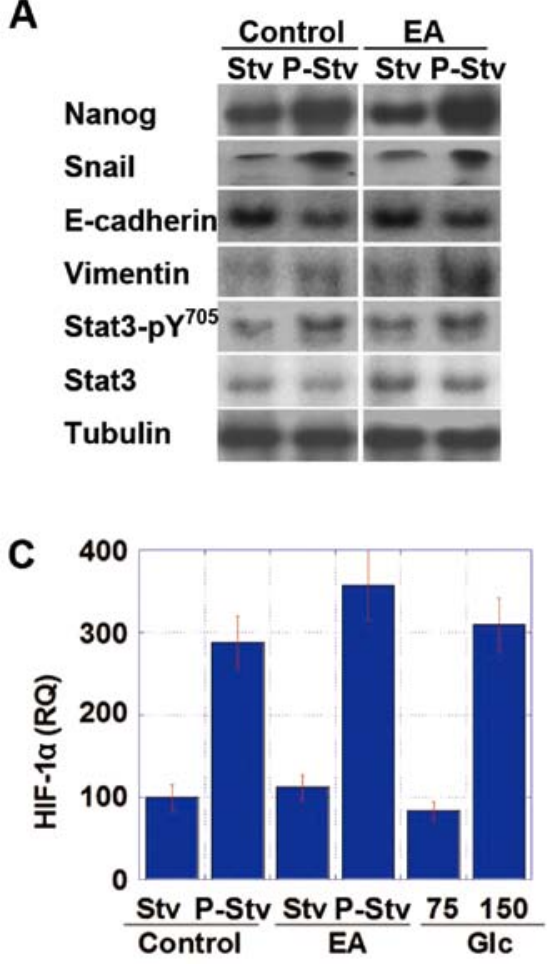

B

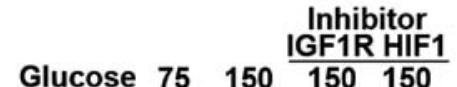

$\begin{array}{llll}\text { Glucose } & 75 \quad 150 \quad 150 \quad 150\end{array}$
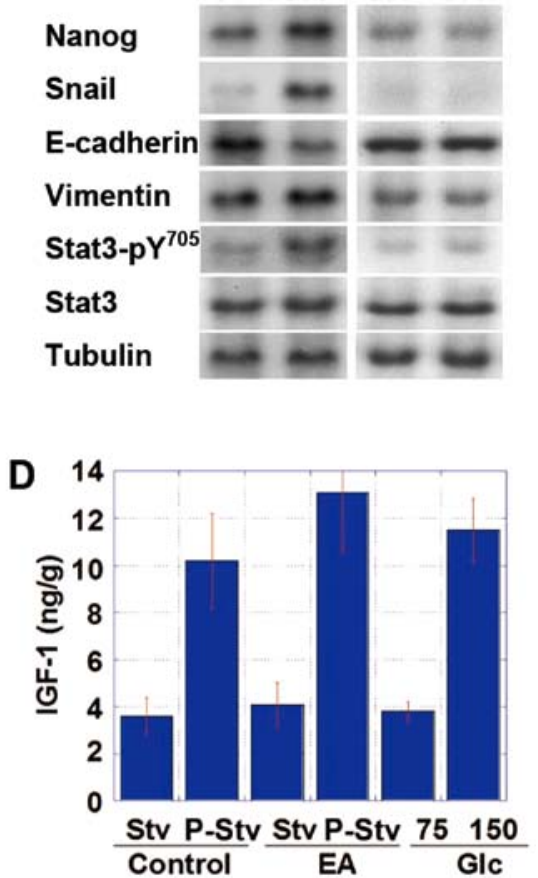

Figure 6. Effect of post-starvation overconsumption on the epithelial-mesenchymal transition (EMT) phenotype of CT26 cells. (A) Protein levels of stemness- and EMT-associated factors were examined in a mouse model of CT26 cell tumors of ICR-mice fed the control or elaidic acid (EA)-enriched diet under starvation (Stv) and post-starvation (over-intake) (P-Stv) conditions. The time schedule was the same as that shown in Fig. 5. (B) Protein levels of stemness- and EMT-associated factors in in vitro experiment of CT26 cells following simulation of the starvation-over-intake cycle. CT26 cells were treated with hypoglycemic DMEM ( $75 \mathrm{mg} / \mathrm{dl}$ glucose) for $24 \mathrm{~h}$, followed by normoglycemic DMEM (150 mg/dl glucose) for $24 \mathrm{~h}$, and then analyzed. For the examination of the signaling pathway, the cells were treated with inhibitors of insulin-like growth factor-1 receptor (IGF-1R) or hypoxia-induced factor- $1 \alpha$ (HIF-1 $\alpha$ ) during cultivation in the presence of each glucose concentration. (C and D) Expression of (C) HIF-1 $\alpha$ mRNA and (D) IGF-1 protein in the intermittent calorie restriction (ICR)-model or in CT26 cells cultured in 75/100 mg/dl glucose. RQ, relative quantity (\%).

exhibited no suppression of tumor growth, compared with those receiving a normal diet (group CNT). In the context of an EA-enriched diet, CCR may therefore not be effective for tumor suppression; however, CCR did not enhance tumor growth. The animals subjected to CCR also exhibited no increase in lung micrometastasis in comparison to the mice in the ICR group. These findings suggest that CCR may not affect cancer growth or metastasis.

Mice receiving either an EA-enriched diet or the control diet with ICR exhibited marked increases in lung micrometastasis, compared to the control animals. Likewise, both groups of mice exhibited surges in the mRNA expression of stemnessassociated genes within the blood. These findings suggest that the release of CSCs into the blood may be associated with the starvation-overeating cycle.

To assess the mechanisms underlying the effect of poststarvation food overconsumption on metastasis, we compared energy production, stemness and EMT phenotype in CT26 cells both in vivo and in vitro. From the starvation phase to the post-starvation phase, blood sugar concentrations were increased 2-fold in mouse tumors in vivo. The transition from the low- to high-glucose conditions altered the primary energy production pathway from the OXPHOS pathway to GL/LF, which is compatible with the Crabtree effect. The Crabtree effect (26) is associated with an increase in stemness and acquisition of the EMT phenotype through the IGF-1/HIF1- $\alpha /$ Nanog/Stat3 signaling pathway, and HIF-1 $\alpha$ expression is upregulated by hyperglycemia (27). IGF-1 and AKT are known as the non-hypoxic HIF-1 $\alpha$ induction pathway (28). In particular, the hypoglycemic-hyperglycemic transition promotes IGF-1 expression $(29,30)$, and AKT is located downstream of IGF-1 in this pathway (31). Moreover, HIF- $1 \alpha$ is a key factor necessary for switching the energy production pathway from OXPHOS to GL/LF through the activation of pyruvate dehydrogenase $(32,33)$. This switching induces higher levels of side population, and promotes the expression of Nanog and Oct3/4 (34), which in turn activates stat3, resulting in the induction of Snail and a subsequent EMT phenotype $(35,36)$. Thus, the Crabtree effect, a temporal increment of the Warburg effect, may be closely associated with the acquisition of metastatic potential in cancer cells.

EA is a main component of industrial dietary TFAs, which play an important role in the progression and metastasis of cancer, as well as in cardiovascular diseases $(8,37)$. The consumption of TFAs is associated with overeating behaviors (38), which may result in a preference for fast food or snacks containing TFAs (39). In this study, we focused on the intake of EA under conditions of ICR. Such conditions can be generalized to the intake of cancer-promoting compounds accompanied by intermittent overeating.

Our data indicated that ICR led to a starvation-overeating cycle. Intermittent feeding increases the expression of orexigenic neurotransmitters, agouti-related peptide and neuropeptide Y. In addition, ICR inhibits the expression of 
pro-opiomelanocortin in the hypothalamus of rodents, even on feeding days, thus explaining the overeating pattern observed in this study $(40,41)$. Feeding uncertainty may induce 'food addiction', particularly to fat- and sugar-rich diets (42). This conclusion is supported by the weight loss exhibited by the control diet group subjected to ICR, but not by the high-fat and EA diet groups subjected to ICR in this study.

In conclusion, overeating associated with ICR abrogated $\mathrm{CR}$ and weight reduction in our models. Moreover, overeating after ICR induced the Crabtree effect in cancer cells, resulting in increased numbers of CSCs and cancer cells circulating within the blood. The Warburg effect or glycolytic energy production is linked closely with malignant phenotypes, such as metastasis $(43,44)$. Our data on ICR are compatible with the mechanism. We also demonstrated that dietary EA was associated with an increment in the metastatic potential of tumors by the acquisition of the EMT phenotype. We have already previously reported the mechanism of EA-induced EMT with the enhancement of stemness (23). Restriction-associated overeating may be the result of an instinctive behavior. Our observations suggest that an irregular dietary intake evoking the starvation-overeating cycle may endow cancer cells with metastatic ability.

\section{Acknowledgements}

The authors would like to thank Ms. Tomomi Masutani for her expert assistance with the preparation of this manuscript. This study was supported by MEXT KAKENHI grant nos. 16H05164, 17K15648, 17K19923 and 16K19087.

\section{References}

1. Lee $\mathrm{C}$ and Longo V: Dietary restriction with and without caloric restriction for healthy aging. F1000Res 5: 5, 2016.

2. Allott EH and Hursting SD: Obesity and cancer: Mechanistic insights from transdisciplinary studies. Endocr Relat Cancer 22: R365-R386, 2015.

3. Barnosky AR, Hoddy KK, Unterman TG and Varady KA Intermittent fasting vs daily calorie restriction for type 2 diabetes prevention: A review of human findings. Transl Res 164: 302-311, 2014.

4. Varady KA: Intermittent versus daily calorie restriction: Which diet regimen is more effective for weight loss? Obes Rev 12 : e593-e601, 2011.

5. Johnstone A: Fasting for weight loss: An effective strategy or latest dieting trend? Int J Obes 39: 727-733, 2015.

6. Lv M, Zhu X, Wang H, Wang F and Guan W: Roles of caloric restriction, ketogenic diet and intermittent fasting during initiation, progression and metastasis of cancer in animal models: A systematic review and meta-analysis. PLoS One 9: e115147, 2014.

7. Thompson HJ and McTiernan A: Weight cycling and cancer: Weighing the evidence of intermittent caloric restriction and cancer risk. Cancer Prev Res (Phila) 4: 1736-1742, 2011.

8. Calder PC: Functional roles of fatty acids and their effects on human health. JPEN J Parenter Enteral Nutr 39 (Suppl 1): 18S-32S, 2015.

9. Rose DP: Dietary fatty acids and cancer. Am J Clin Nutr 66 (Suppl 4): 998S-1003S, 1997.

10. Kuniyasu H, Ohmori H and Fujii K: Significance of trans fatty acids in colorectal cancer. In: Current Research in Cancer. Vol. 9. Sunitha M (ed). Research Media, Kerala, India, pp21-29, 2015.

11. Food and Drug Administration: Final Determination Regarding Partially Hydrogenated Oils. 2015.

12. Cascio G, Schiera G and Di Liegro I: Dietary fatty acids in metabolic syndrome, diabetes and cardiovascular diseases. Curr Diabetes Rev 8: 2-17, 2012.
13. Mozaffarian D, Aro A and Willett WC: Health effects of transfatty acids: Experimental and observational evidence. Eur J Clin Nutr 63 (Suppl 2): S5-S21, 2009.

14. Zong WX, Rabinowitz JD and White E: Mitochondria and Cancer. Mol Cell 61: 667-676, 2016.

15. Warburg O: On respiratory impairment in cancer cells Science 124: 269-270, 1956.

16. Kellenberger LD, Bruin JE, Greenaway J, Campbell NE, Moorehead RA, Holloway AC and Petrik J: The role of dysregulated glucose metabolism in epithelial ovarian cancer. J Oncol 2010: 514310, 2010.

17. Dell' Antone P: Energy metabolism in cancer cells: How to explain the Warburg and Crabtree effects? Med Hypotheses 79: 388-392, 2012.

18. Kuwada M, Chihara Y, Luo Y, Li X, Nishiguchi Y, Fujiwara R, Sasaki T,FujiiK,OhmoriH,Fujimoto K, etal:Pro-chemotherapeutic effects of antibody against extracellular domain of claudin-4 in bladder cancer. Cancer Lett 369: 212-221, 2015.

19. Livak KJ and Schmittgen TD: Analysis of relative gene expression data using real-time quantitative PCR and the 2(-Delta Delta C(T)) method. Methods 25: 402-408, 2001.

20. Kuniyasu H, Yano S, Sasaki T, Sasahira T, Sone S and Ohmori H: Colon cancer cell-derived high mobility group 1/amphoterin induces growth inhibition and apoptosis in macrophages. Am J Pathol 166: 751-760, 2005.

21. Kuniyasu H, Oue N, Wakikawa A, Shigeishi H, Matsutani N, Kuraoka K, Ito R, Yokozaki H and Yasui W: Expression of receptors for advanced glycation end-products (RAGE) is closely associated with the invasive and metastatic activity of gastric cancer. J Pathol 196: 163-170, 2002.

22. Ohmori H, Fujii K, Kadochi Y, Mori S, Nishiguchi Y, Fujiwara R, Kishi S, Sasaki T and Kuniyasu H: Elaidic acid, a trans-fatty acid, enhances the metastasis of colorectal cancer cells. Pathobiology 84 : 144-151, 2017

23. Fujii K, Luo Y, Fujiwara-Tani R, Kishi S, He S, Yang S, Sasaki T, Ohmori $\mathrm{H}$ and Kuniyasu H: Pro-metastatic intracellular signaling of the elaidic trans fatty acid. Int J Oncol 50: 85-92, 2017.

24. Hatori M, Vollmers C, Zarrinpar A, DiTacchio L, Bushong EA, Gill S, Leblanc M, Chaix A, Joens M, Fitzpatrick JA, et al: Timerestricted feeding without reducing caloric intake prevents metabolic diseases in mice fed a high-fat diet. Cell Metab 15: 848-860, 2012.

25. Zarrinpar A, Chaix A and Panda S: Daily eating patterns and their impact on health and disease. Trends Endocrinol Metab 27: 69-83, 2016.

26. Crabtree HG: Observations on the carbohydrate metabolism of tumours. Biochem J 23: 536-545, 1929.

27. Wang Y, Zhu YD, Gui Q, Wang XD and Zhu YX: Glucagoninduced angiogenesis and tumor growth through the HIF-1-VEGF-dependent pathway in hyperglycemic nude mice. Genet Mol Res 13: 7173-7183, 2014.

28. Xu Q, Zhang Q, Ishida Y, Hajjar S, Tang X, Shi H, Dang CV and Le AD: EGF induces epithelial-mesenchymal transition and cancer stem-like cell properties in human oral cancer cells via promoting Warburg effect. Oncotarget 8: 9557-9571, 2017.

29. Lee $C$ and Longo VD: Fasting vs dietary restriction in cellular protection and cancer treatment: From model organisms to patients. Oncogene 30: 3305-3316, 2011.

30. Guimarães TA, Farias LC, Santos ES, de Carvalho Fraga CA, Orsini LA, de Freitas Teles L, Feltenberger JD, de Jesus SF, de Souza MG, Santos SH, et al: Metformin increases PDH and suppresses HIF-1 $\alpha$ under hypoxic conditions and induces cell death in oral squamous cell carcinoma. Oncotarget 7: 55057-55068, 2016.

31. Klement RJ and Champ CE: Calories, carbohydrates, and cancer therapy with radiation: Exploiting the five R's through dietary manipulation. Cancer Metastasis Rev 33: 217-229, 2014.

32. Shaw RJ: Glucose metabolism and cancer. Curr Opin Cell Biol 18: 598-608, 2006.

33. Garcia-Heredia JM and Carnero A: Decoding Warburg's hypothesis: Tumor-related mutations in the mitochondrial respiratory chain. Oncotarget 6: 41582-41599, 2015.

34. Zhong Y, Li X, Yu D, Li X, Li Y, Long Y, Yuan Y, Ji Z, Zhang M, Wen JG, et al: Application of mitochondrial pyruvate carrier blocker UK5099 creates metabolic reprogram and greater stem-like properties in LnCap prostate cancer cells in vitro. Oncotarget 6: 37758-37769, 2015.

35. Yin X, Zhang BH, Zheng SS, Gao DM, Qiu SJ, Wu WZ and Ren ZG: Coexpression of gene Oct4 and Nanog initiates stem cell characteristics in hepatocellular carcinoma and promotes epithelialmesenchymal transition through activation of Stat3-Snail signaling. J Hematol Oncol 8: 23, 2015. 
36. Liu S, Sun J, Cai B, Xi X, Yang L, Zhang Z, Feng Y and Sun Y: NANOG regulates epithelial-mesenchymal transition and chemoresistance through activation of the STAT3 pathway in epithelial ovarian cancer. Tumour Biol 37: 9671-9680, 2016.

37. Ganguly R and Pierce GN: Trans fat involvement in cardiovascular disease. Mol Nutr Food Res 56: 1090-1096, 2012.

38. Prentice AM: Overeating: The health risks. Obes Res 9 (Suppl 4): 234S-238S, 2001.

39. Costa N, Cruz R, Graça P, Breda J and Casal S: Trans fatty acids in the Portuguese food market. Food Control 64: 128-134, 2016.

40. Chausse B, Solon C, Caldeira da Silva CC, Masselli Dos Reis IG, Manchado-Gobatto FB, Gobatto CA, Velloso LA and Kowaltowski AJ: Intermittent fasting induces hypothalamic modifications resulting in low feeding efficiency, low body mass and overeating. Endocrinology 155: 2456-2466, 2014.

41. Lauzurica N, García-García L, Pinto S, Fuentes JA and Delgado M: Changes in NPY and POMC, but not serotonin transporter, following a restricted feeding/repletion protocol in rats. Brain Res 1313: 103-112, 2010.
42. Corwin RL: The face of uncertainty eats. Curr Drug Abuse Rev 4 174-181, 2011.

43. Zhao J, Huang X, Xu Z, Dai J, He H, Zhu Y and Wang H: LDHA promotes tumor metastasis by facilitating epithelial mesenchymal transition in renal cell carcinoma. Mol Med Rep 16: 8335-8344, 2017.

44. Morandi A, Taddei ML, Chiarugi P and Giannoni E: Targeting the metabolic reprogramming that controls epithelial-to-mesenchymal transition in aggressive tumors. Front Oncol 7: 40, 2017.

(C) (1) () This work is licensed under a Creative Commons

COY NO Attribution-NonCommercial-NoDerivatives 4.0 International (CC BY-NC-ND 4.0) License. 